\title{
INCIDENCIA DE FACTORES PERSONALES Y LABORALES EN LOS SALARIOS DEL SECTOR HOSTELERO: UNA VISIÓN DE GÉNERO
}

\author{
Míriam Ons-Cappa \\ Alejandro García-Pozo \\ José Luis Sánchez-Ollero \\ Universidad de Málaga
}

\section{RESUMEN}

Este artículo aporta evidencia empírica sobre las diferencias en los rendimientos salariales desde una perspectiva de género en el sector hostelero español, comparándolas con otros sectores de la economía española. Mediante la estimación de una función minceriana, los resultados obtenidos revelan una significativa diferencia negativa del rendimiento del capital humano en la hostelería frente al resto de sectores y una mayor penalización salarial asociada al rendimiento educativo para el caso de la mujer en la hostelería, en contraposición a lo que ocurre en el resto de sectores analizados.

Palabras clave: capital humano, turismo, hostelería, diferencia salarial, mujer, género.

Impact of personal and labour factors in hospitality wages: a gender view

\begin{abstract}
This article provides empirical evidence on differences in wage income from a gender perspective in the Spanish hospitality sector, as compared with other sectors of the Spanish economy. Using a Mincerian function, the results indicate a significant negative difference in the returns on human capital in the hospitality compared to other sectors and greater wage penalty associated with the educational returns for the case of women in the hospitality industry, in contraposition to what happens in other sectors analyzed.
\end{abstract}

Keywords: human capital, tourism, hospitality, wage differentials, woman, gender.

Recibido: 24 de febrero de 2015

Devuelto para su revisión: 5 de junio de 2015

Aceptado: 3 de octubre de 2015

Departamento de Economía Aplicada (Estructura Económica). Universidad de Málaga. C/ El Ejido, nº 6 . 29071 MÁLAGA (España).E-mail: moc@uma.es, alegarcia@uma.es, jlsanchez@uma.es 


\section{INTRODUCCIÓN}

La relevancia del turismo para España es un hecho constatable estadísticamente. Así lo corrobora, por ejemplo, su aportación del 10,9\% del PIB (Cuenta Satélite del Turismo de España, 2012), su dotación de empleo a un 13,6\% sobre el total nacional de ocupados (Encuesta de Población Activa, EPA, 3er trimestre 2015) o el saldo positivo de 34.923 millones de euros registrado por la rúbrica de turismo y viajes de la Balanza de Pagos en 2015.

Según la Organización Mundial del Turismo (2016), en 2014, España registró 65 millones de llegadas de turistas internacionales (5,74\% sobre un total de 1.133 millones) que generaron un ingreso de 65,2 millones de dólares $(5,24 \%$ sobre un total de 1.245 millones). Estos datos situaron al país en $3^{\mathrm{a}}$ y $2^{\mathrm{a}}$ posición en el ranking de llegadas de turistas internacionales y de ingresos por turismo internacional, respectivamente.

Los datos señalados confirman la trascendencia del turismo para la economía española. Concretamente en lo que se refiere al mercado de trabajo turístico, y a pesar de su importancia en la economía española, se ha caracterizado tradicionalmente por un gran número de trabajos no cualificados, inestabilidad y elevada rotación, salarios relativamente bajos y escasas perspectivas de promoción (Sánchez-Ollero, Campos-Soria y García-Pozo, 2014).

Entre todas las ramas de actividad del sector turístico, la hostelería es la que más importancia tiene en términos de ocupados (Según Turespaña, 64,78\% sobre un total de 2.322.381 ocupados en 2015) y la que mayor proporción de mujeres ocupa (Según Turespaña 769.000 mujeres frente a 736.100 hombres en 2015). Estas trabajadoras, a su vez, se encuentran especialmente afectadas por una segregación horizontal y vertical en los puestos de trabajo (Campos-Soria, Ortega-Aguaza y Ropero-García, 2010), una elevada contratación temporal (Baum, 2013) y unos rendimientos educativos más bajos (GarcíaPozo, Campos-Soria, Sánchez-Ollero y Marchante-Lara, 2012; Lillo Bañuls, 2009). Por lo anterior, la combinación de las características mencionadas del mercado de trabajo en ese sector, favorece la precariedad salarial en la mujer en comparación con el hombre, generando lo que se conoce como brecha salarial de género. Este fenómeno, también denominado diferencia o desigualdad salarial y, comúnmente confundido con la discriminación salarial, se emplea para conceptualizar la distancia existente entre el salario masculino y femenino. Por su parte, la discriminación salarial hace alusión a la parte de la diferencia salarial que se fundamenta en motivos estrictamente discriminatorios, es decir, que no se puede justificar por razones diferentes al sexo de la persona (De Cabo y Garzón, 2007).

Desde los inicios de la Unión Europea, el principio de igualdad retributiva entre hombres y mujeres por un trabajo de igual valor, ha sido una preocupación constante. De hecho, este principio se postula por primera vez en el art. 119 del Tratado de Roma en el año 1957. A pesar de ello, parece que los esfuerzos aún no se concretan en una reducción de esa desigualdad retributiva. De acuerdo con Eurostat (2016), en 2014, el promedio de la brecha salarial de género se cifró en un 16,1\% para el conjunto de los Estados Miembros (EU-28). Esto significa que el salario bruto medio por hora de la mujer era un $16,1 \%$ inferior al del hombre. La proclividad de la mujer a ser contratada a tiempo parcial, realizar mayores descansos en sus carreras profesionales, trabajar en industrias de bajas 
remuneraciones y en sectores con menor poder de negociación, son algunas de las razones que justifican parte del desajuste señalado (Ponzellini, Aumayr, \& Wolf, 2010).

Como han puesto de manifiesto De Cabo y Garzón (2007), son prolíficos los estudios a nivel internacional y nacional que se han ocupado en cuantificar, explicar y confirmar que el salario medio de los hombres es, por término medio, superior al que perciben las mujeres. No obstante, hay que partir de la consideración de la metodología empleada para tal fin. En ese sentido, en el estudio de la brecha salarial entre hombres y mujeres se diferencian dos aplicaciones metodológicas por excelencia: por un lado, la que trata de explicar las diferencias a partir del rendimiento salarial generado por el capital humano de los trabajadores (Mincer, 1974); y, de otro lado, aquélla que incorpora el factor de la discriminación para completar la explicación de dicha desigualdad (Blinder, 1973; Oaxaca, 1973).

El objetivo principal de este trabajo es analizar la incidencia de una serie de factores personales y laborales sobre el salario de los trabajadores/as del subsector de la hostelería española, comparándolos con otros sectores de actividad, todo ello desde una perspectiva de género. Para ello, se formula una variación de la función de salarios de Mincer (1974), en la medida en que ésta se define como una regresión lineal, en lugar de la cuadrática original, introduciéndose a su vez variables adicionales en el modelo que pretenden recoger otros factores que podrían condicionar el rendimiento salarial.

Para la consecución del objetivo planteado, el resto de este documento se ha organizado de la siguiente manera: en el apartado 2, se profundiza sucintamente en la literatura sobre las diferencias salariales entre hombres y mujeres en el sector turístico. A continuación, en el apartado 3, se realiza la exposición metodológica, selección y descripción de las variables. En el epígrafe 4, se presentan los datos y se lleva a cabo un breve análisis descriptivo. En el apartado 5, se exponen los principales resultados obtenidos. Finalmente, se presentan las conclusiones obtenidas y las recomendaciones de política económica propuestas.

\section{REVISIÓN DE LA LITERATURA}

El origen del estudio de las diferencias salariales de género data de mediados del siglo XIX. Fue John Stuart Mill, en su obra Principios de Economía Política (1848), uno de los primeros economistas en apuntar la desigualad de los salarios de las mujeres frente al de los hombres.

No obstante, la medición del alcance de este fenómeno no comenzaría hasta casi un siglo más tarde, con los primeros trabajos de Becker (1964), que fueron completados posteriormente con la propuesta metodológica de Blinder (1973) y Oaxaca (1973). Desde entonces, como ya se ha señalado, la diferencia y discriminación salarial por razón de sexo se ha venido explicando, por un lado, desde la Teoría del Capital Humano y, por otro, desde la óptica de la Teoría de la discriminación.

La Teoría del Capital Humano (Becker, 1964; Mincer, 1974; Schultz, 1961) ha sido ampliamente utilizada tanto teórica como empíricamente para analizar las diferencias salariales entre hombres y mujeres. En síntesis, esta teoría postula, bajo la presunción de racionalidad de los individuos, que éstos invierten en capital humano para incre- 
mentar sus habilidades y/o capacidades, en función de los costes directos e indirectos en los que tengan que incurrir y los beneficios que les vaya a reportar. Enmarcada en la corriente neoclásica, para la que los factores de producción, en este caso el trabajo de los individuos, son retribuidos según su productividad, los incrementos en los rendimientos marginales se traducen en aumentos en los salarios (Selva Sevilla, 2004). En otras palabras, "las diferencias en capital humano entre hombres y mujeres generan sus diferencias en productividad. Y dado que los salarios están ligados a la productividad, esa es la causa de las diferencias salariales observadas" (Ramos, Rey-Maquieira y Tugores, 2002: 240).

Ahora bien, las razones teóricas que explican dichas diferencias de inversión se ven influenciadas por factores sociológicos y culturales. En este sentido, las mujeres acumulan menos capital humano debido, entre otras cosas, a su mayor responsabilidad y dedicación al cuidado de la familia y el hogar. En consecuencia, cuentan con una menor experiencia laboral y un mayor condicionamiento en la elección del puesto de trabajo, en tanto en cuanto, eligen ocupaciones en las que, en términos relativos, la remuneración inicial es alta, el rendimiento de la experiencia bajo, y el perjuicio derivado de la retirada temporal de la vida activa, ligero (Anker, 1997).

Por lo que respecta a la teoría de la discriminación o modelo del "gusto por la discriminación”, Becker (1957) la concibe como la preferencia del discriminador, en este caso el empresario, de no contratar a mujeres (McConnel \& Brue, 1996). Desde este enfoque, no sólo se acepta que a distintos niveles de productividad le corresponden diferentes salarios, sino que en función de la pertenencia a un grupo determinado (ser mujer, en este caso), dicha productividad puede verse remunerada de diferente manera (Rueda Narváez, 2010). En esta línea interpretativa, podemos encontrar autores como Thurow (1975) cuya reflexión se basa en que los empresarios, buscando alcanzar la máxima rentabilidad, invertirán en la formación de aquellos trabajadores que tienen mayor probabilidad de permanecer más tiempo en la empresa, esto es, en los hombres.

Posteriormente, surgen otros planteamientos para explicar las diferencias salariales entre hombres y mujeres. Por su parte, Lundberg \& Starz (1983), tratan de racionalizar las desigualdades de género basándose en la presencia de información asimétrica. Así, los empresarios en su desconocimiento de los niveles de productividad de los potenciales trabajadores, tratan de estimarlos a partir de distintas "señales". Estos autores concluyen que una señal menos fiable para el caso del grupo minoritario (mujeres) puede explicar una menor oferta salarial. Otros autores como Blau (1972) o Malkiel \& Malkiel (1973), señalan la segregación ocupacional como factor determinante y explicativo de las diferencias salariales entre hombres y mujeres en el mercado laboral.

Todos estos planteamientos diferentes se han ido conjugando con el transcurso del tiempo, llegando a la conclusión unánime de que una parte de la diferencia salarial por razón de sexo está explicada por las diferencias en las dotaciones de capital humano que presentan hombres y mujeres. Sin embargo, existe otra parte de la mencionada brecha salarial que no puede explicarse por razones diferentes al sexo (De Cabo Serrano y Garzón, 2007).

Ya en la década de los 90, Wilkinson \& Pratiwi (1995) advirtieron la exigua literatura sobre la relación entre turismo y género. Estos autores junto con Kinnard \& Hall (1994), 
Sinclair (1997) y Byrne Swain (1995) fueron pioneros en analizar las cuestiones de género en el sector turístico. En sus trabajos, se afronta la tarea de definir el marco conceptual del análisis del género en esta actividad y se presentan una serie de casos de estudio que analizan, desde distintas perspectivas, los efectos del desarrollo turístico (Ramos, ReyMaquieira y Tugores, 2002).

En referencia concreta a autores que tratan las diferencias salariales entre hombres y mujeres en la industria turística, podemos citar, entre los primeros, a Lee \& Kang (1998), quienes estiman el grado de desigualdad de los ingresos de los trabajadores del sector turístico de Corea del Sur. Asimismo, destaca el trabajo de Sparrowe \& Iverson (1999) para el sector hostelero de Estados Unidos. En él se presenta, por primera vez, un modelo multivariante que permite explicar los efectos del género en el contexto de otros factores que también afectan a los ingresos. Y, además, estiman ecuaciones de ingresos por ocupación. Entre los últimos estudios publicados sobre esta materia, se encuentran el firmado por Fleming (2015) para el sector de la hostelería estadounidense y el de Ferreira Freire Guimaraes y Silva (2016) para el sector turístico de Brasil.

En España, si bien es cierto que el tema de la diferencia y/o discriminación salarial de género ha sido muy prolífico (De la Rica y Ugidos, 1995; Hernández Martínez, 1995; Moltó, 1984; Peinado, 1988; Prieto, 1995; Ribaud y Hernández, 1989; Simón, 2006; Ulibarri Arce, 2003), su desarrollo ha sido tardío por la falta de estadísticas adecuadas. Por tanto, cabría esperar que los estudios sobre la temática referenciada en el sector turístico español hayan seguido una tendencia similar. Es importante destacar el trabajo seminal en este campo de Lillo Bañuls y Ramón Rodríguez (2005) donde, a partir de ecuaciones de ingresos, se analizan las diferencias en los rendimientos educativos (y otras variables) entre hombres y mujeres en el sector turístico español y sus repercusiones en los salarios. En esta misma línea de investigación sobre el capital humano del sector turístico español, también son destacables los trabajos posteriores de estos autores (Lillo Bañuls, 2009; Lillo Bañuls y Casado Díaz, 2010, 2011, 2012) . Otra aportación reseñable es la de Muñoz-Bullón (2009), en la que se analiza la discriminación salarial entre hombres y mujeres en la industria turística española en comparación con otros sectores.

Asimismo, son destacables otros estudios cuya atención se centra en diferentes aspectos para explicar y/o evidenciar las diferencias salariales de género. Así, podemos citar los trabajos sobre segregación ocupacional (Campos-Soria et al., 2010; Campos-Soria, Marchante-Mera y Ropero-García, 2011; Campos-Soria, Ortega-Aguaza y Ropero-García, 2009; Ramos et al., 2002) desajuste educativo (Marchante, Ortega y Pagán, 2005; Marchante Mera, Ortega Aguaza y Sánchez Ollero, 2004; Marrero Rodríguez, 2015); diferencias regionales (García-Pozo et al., 2012); o distribución salarial (Casado-Díaz y Simón, 2016).

En este trabajo, y siguiendo la Teoría del capital humano, se analizan y comparan las diferencias salariales entre hombres y mujeres para cuatro sectores de la economía española: Industria, Construcción, Hostelería y el resto de servicios sin incluir la hostelería, con la intención de verificar una situación de discriminación salarial de las mujeres en la hostelería. 


\section{METODOLOGíA}

Con datos procedentes de la Encuesta de Estructura Salarial 2010, con este trabajo se realizan tanto análisis descriptivos que permitan tener una visión de conjunto de este sector y un elemento de comparación con otros sectores de actividad, como análisis econométricos que nos permitan estimar la incidencia cuantitativa de los factores personales y laborales sobre el salario de los trabajadores y trabajadoras de la hostelería en España, como ya se ha señalado.

Para esta segunda tarea, se estima la función salarial de Mincer (1974) a la que se incorporan otras variables no relacionadas directamente con el capital humano sobre el salario de las trabajadores/as del sector analizado. La ecuación de salario aquí propuesta difiere de la original de Mincer porque utiliza una especificación lineal para las variables experiencia previa y antigüedad (García-Pozo et al., 2012). Esta opción se ha elegido debido a la elevada multicolinealidad ${ }^{1}$ entre cada una de estas variables y su cuadrado. Dado que los rendimientos de la experiencia previa y de la antigüedad se presentan en etapas tardías de la vida laboral, el cálculo a mitad de la carrera profesional no debería verse muy afectado con este procedimiento alternativo de estimación (Strauss \& Maisonneuve, 2007). De esta forma, la función de salarios que se pretende estimar, tanto para hombres como para mujeres y para el sector de la hostelería y del resto de sectores sería de la forma:

$$
\begin{gathered}
\log (W r)=\beta_{0}+\beta_{1} S+\beta_{2} \text { Exper }+\beta_{3} \text { Anti }+\beta_{4} \text { Ame_Latin }+\beta_{5} \text { Indef_TC }+\beta_{6} T_{-} \text {Emp }+ \\
\beta_{7} \text { Sobreedu }+\beta_{8} \text { Infraedu }+\varepsilon_{\mathrm{i}}[1]
\end{gathered}
$$

La variable dependiente es el salario real bruto por hora en logaritmos $(\mathrm{Wr})$ calculado en base a la información recogida en la EES-2010; en el lado derecho de la ecuación, además de la constante, se consideran las características de los trabajadores y también de los puestos de trabajo. La constante, $\beta_{0}$, es el término cuyo valor indica la parte del salario que no está afectada por las variables independientes, sino por otras (estilo de vida, clima...) que no se han considerado para la especificación de este trabajo.

En el grupo de las variables de capital humano, se consideran los años medios de estudio (S), la experiencia previa (Exper), la antigüedad en la empresa (Antig), y así como una variable ficticia de carácter personal representativa de la nacionalidad latinoamericana del trabajador (Ame_Latin). Se ha valorado de interés la inclusión de esta variable dada la representatividad de la población extranjera latinoamericana trabajadora en el subsector de la hostelería española (casi el 50\% sobre el total de 298.315 inmigrantes según la Encuesta Nacional de Inmigrantes en 2007).

Con respecto a las características del puesto de trabajo, se definen las siguientes variables ficticias (dummy): contrato indefinido a tiempo completo (Indef_TC), el tamaño de

1 En todas las estimaciones planteadas los valores del coeficiente de correlación entre las variables señaladas y sus cuadrados superaba el valor de 0.917 , alcanzando el valor máximo en la estimación correspondiente a mujeres en el sector de la hostelería para la variable Experiencia previa (0.952). Por esta razón se optó por la especificación lineal. 
la empresa ( $\left.T_{-} E m p\right)$ y la educación formal del trabajador en relación a los requerimientos del puesto de trabajo: sobreeducado (Sobreedu) e infraeducado (Infraedu). Por último, $\varepsilon$ es el término de perturbación aleatoria.

\subsection{Descripción de variables}

A continuación, se procede a la descripción de cada una de las variables empleadas en la ecuación salarial.

Para la variable años medios de estudio (S), la Encuesta de Estructura Salarial 2010, proporciona información sobre el nivel educativo más alto completado por los individuos. A cada uno de los niveles educativos se les asigna un valor numérico aproximado al número de años que se estiman necesarios para completar los estudios correspondientes a cada nivel. Por tanto, los valores de las variables son los siguientes:

- 2 años para los analfabetos o sin estudios.

- 5 años para individuos con educación primaria.

- 8 años para los que completan el primer ciclo de educación secundaria.

- 9 y 11 años en individuos que finalicen primer y segundo ciclo de formación profesional y equivalentes.

- 12 años para trabajadores con el segundo ciclo de educación secundaria completado.

- 15 años para diplomados universitarios.

- 17 años para licenciados y con estudios de postgrado.

Para la definición de las variables experiencia previa (Exp) y antigüedad en la empresa (Antig) se ha seguido a Arrazola Vacas y Hevia Payá (2006). En relación con la duración de la vida laboral del individuo, debe considerarse lo siguiente: 1) por cuestiones legales, los individuos no trabajan antes de los 16 años; 2) se presupone la incompatibilidad de trabajar y estudiar simultáneamente; 3) y la edad legal de jubilación se eleva a 65 años para los individuos que forman parte de la muestra.

La variable contrato indefinido y a tiempo completo (Indef_TC) toma el valor 1 cuando el trabajador desarrolla su labor durante una jornada laboral completa (40 horas semanales) y por tiempo indefinido y, 0 en los demás casos. Con ello se pretende valorar si este tipo de contrato conlleva prima salarial, con relación a los trabajadores con contrato temporal o a tiempo parcial. La agregación en una sola variable de ambos tipos de contrato obedece a que suelen utilizarse en el sector para facilitar la flexibilidad numérica de la plantilla (Nickson, 2007) entendida como la capacidad de los empleadores para ajustar la oferta de trabajo a las fluctuaciones de la demanda de sus productos y servicios; y suele implicar mayor precariedad en el empleo, menor salario, ausencia de oportunidades de formación y escasas posibilidades de promoción profesional para los trabajadores.

La variable tamaño empresarial (T_Emp), toma el valor 1 cuando el empleado trabaja en una empresa de más de 19 asalariados, y 0 en el resto de los casos. De esta forma se pretende recoger el efecto de los pequeños establecimientos, como bares, restaurantes y pequeños hoteles en el caso de la hostelería, sobre los salarios de los trabajadores y trabajadoras. 
Mediante las variables Sobreedu e Infraedu se trata de analizar el desajuste educativo de los trabajadores/as de la muestra. Por desajuste educativo se entiende la discrepancia entre el nivel educativo alcanzado por el individuo y el requerido por su puesto de trabajo. Bajo esta perspectiva, los trabajadores pueden estar sobreeducados, adecuadamente educados o infraeducados, si su nivel de educación está por encima, es igual o queda por debajo, respectivamente, del nivel educativo requerido por el puesto de trabajo. En la literatura se han utilizado diferentes métodos ${ }^{2}$ para la medición del desajuste educativo, agrupados en objetivos, subjetivos y estadísticos. El método empleado en este estudio es el estadístico, que calcula la educación requerida para un trabajador en función del nivel educativo medio en cada una de las ocupaciones. En ese sentido, un trabajador está sobreeducado cuando sus años de educación formal superan en una desviación típica la media de los años de la ocupación que desempeña (Verdugo y Verdugo, 1989). En este trabajo, en lugar del estadístico de la media, se sigue la propuesta de Kiker, Santos y Mendes de Oliveiria (1997) y se emplea la moda. De esta manera, son trabajadores adecuadamente educados aquéllos que obtienen un nivel educativo correspondiente a la moda (el más común para el puesto de trabajo analizado) o su contiguo (ya sea el inmediatamente inferior o superior) ${ }^{3}$. Por su parte, la inclusión de las variables representativas de los trabajadores sobreeducados e infraeducados en la función de salarios propuesta en este trabajo, se ha realizado mediante dos variables dummys. Así, se ha definido la componente Sobreedu para recoger a los individuos sobreeducados. Ésta tomará el valor 1 si el trabajador ha alcanzado un nivel superior al representado por la moda de cada ocupación (o mayor al más elevado en el caso de haber considerado dos niveles educativos contiguos); y 0 en el resto de los casos. Y la variable Infraedu, en representación de los individuos infraeducados, tomará el valor 1 si el trabajador ha alcanzado un nivel inferior al representado por la moda de la ocupación (o menor al que ha requerido menos años de estudio en el caso de haber considerado dos niveles educativos contiguos); y 0 en el resto de los casos.

La evidencia empírica disponible permite esperar que los coeficientes estimados de las variables años medios de estudios $(S)$, experiencia previa (Exper), antigüedad en la empresa (Antig), contrato de trabajo indefinido y a tiempo completo (Indef_TC), infraeducados (Infraedu) y tamaño empresarial (T_Emp), tengan un efecto positivo sobre el salario real por hora. En contra, se espera un signo negativo para los coeficientes estimados de las variables América Latina (Ame_Latin) y sobreeducados (Sobreedu).

2 Según Blanco (1997), el método objetivo elabora una clasificación de puestos de trabajo en la que se determina el nivel educativo que se considera necesario para su desempeño y se compara con el del individuo analizado. Por su parte, el método subjetivo se basa en datos obtenidos a partir de encuestas realizadas a trabajadores, sobre sus características personales y las del puesto que ocupan.

3 El procedimiento se amplía en los casos en los que el nivel educativo completado represente un porcentaje inferior al $40 \%$ del total de asalariados dentro de cada ocupación. De este modo, si en una determinada ocupación los años medios de estudios requeridos son 15 (diplomados universitarios), pero la moda incluye un reducido número de casos (menor del 40\%), también se considerarían adecuadamente educados a los trabajadores con el segundo ciclo de educación secundaria (12 años de estudio) o a los licenciados universitarios (17 años). La elección del nivel de estudios inmediatamente inferior o superior a la moda se ha realizado teniendo en cuenta cuál de ellos representa un mayor porcentaje de trabajadores en cada ocupación. 


\section{DATOS Y ANÁLISIS DESCRIPTIVO}

La base de datos utilizada para este trabajo procede de la Encuesta de Estructura Salarial de 2010 (en adelante, EES-2010). Esta encuesta, elaborada por el Instituto Nacional de Estadística, es una investigación sobre la estructura y distribución de los salarios españoles de periodicidad cuatrienal, realizada en el marco de la Unión Europea con criterios comunes de metodología y contenidos.

El sistema de clasificación empleado por la EES-2010 para su elaboración se fundamenta a nivel sectorial, en la Clasificación Nacional de Actividades Económicas CNAE2009 y, a nivel de ocupaciones, en la Clasificación Nacional de Ocupaciones CNO-2011.

El presente trabajo de investigación considera los grupos correspondientes a Industria (E), Construcción (F), Hostelería (I) y Otros servicios $^{5}$ (contiene todos los grupos desde G hasta U, descartando las actividades del grupo I, ya incluidas en el sector de la hostelería). Así, la base de datos aporta información para un total de 46.328 observaciones para la Industria, 11.965 para la Construcción, 6.059 para la Hostelería y 108.498 para Otros servicios.

La hostelería es el sector de referencia, a partir del cual se desprende el siguiente análisis descriptivo de las variables empleadas en el análisis econométrico desarrollado en este trabajo. Los valores medios de las variables analizadas por sector económico y por sexo, para el año 2010, se presentan en la Tabla 1.

\section{Tabla 1}

\section{CARACTERÍSTICAS OBSERVADAS EN LA MUESTRA DE SECTORES SELECCIONADOS}

\begin{tabular}{|c|c|c|c|c|c|c|c|c|c|c|}
\hline \multirow{3}{*}{ Variables } & & \multicolumn{9}{|c|}{ Grupos de actividades CNAE-2009 } \\
\hline & \multicolumn{2}{|c|}{ Industria } & \multicolumn{2}{|c|}{ Construcción } & \multicolumn{2}{|c|}{ Hostelería } & \multicolumn{2}{|c|}{ Otros servicios } & \multicolumn{2}{|c|}{$\begin{array}{c}\text { Media } \\
\text { Nacional }\end{array}$} \\
\hline & $H$ & $M$ & $H$ & $M$ & $H$ & $M$ & $H$ & $M$ & $H$ & $M$ \\
\hline \multirow{2}{*}{$\begin{array}{c}\text { Salario bruto } \\
\text { medio por } \\
\text { hora }\end{array}$} & 13,53 & 10,31 & 10,41 & 9,97 & 10,09 & 7,95 & 13,85 & 10,79 & 13,26 & 10,56 \\
\hline & $(9,08)$ & $(6,43)$ & $(5,78)$ & $(4,84)$ & $(6,88)$ & $(3,59)$ & $(10,48)$ & $(7,00)$ & $(9,56)$ & $(6,79)$ \\
\hline \multirow{2}{*}{$\begin{array}{l}\text { Años medios } \\
\text { de estudio }\end{array}$} & 9,17 & 9,92 & 7,86 & 11,93 & 8,27 & 8,17 & 11,04 & 11,19 & 9,96 & 10,86 \\
\hline & $(3,81)$ & $(4,13)$ & $(3,75)$ & $(3,90)$ & $(3,53)$ & $(3,35)$ & $(4,26)$ & $(4,18)$ & $(4,20)$ & $(4,20)$ \\
\hline \multirow{2}{*}{$\begin{array}{c}\text { Experiencia } \\
\text { previa }\end{array}$} & 13,33 & 12,87 & 18,58 & 12,40 & 15,38 & 18,59 & 13,15 & 13,40 & 13,86 & 13,55 \\
\hline & $(9,35)$ & $(9,66)$ & $(10,58)$ & $(8,63)$ & $(9,88)$ & $(10,70)$ & $(9,52)$ & $(9,97)$ & $(9,77)$ & $(10,01)$ \\
\hline \multirow{2}{*}{$\begin{array}{c}\text { Antigüedad } \\
\text { en el puesto } \\
\text { de trabajo }\end{array}$} & 12,30 & $\mathbf{9 , 9 9}$ & 5,44 & 5,94 & 7,76 & 5,39 & 9,76 & 8,06 & 10,15 & 8,19 \\
\hline & $(11,00)$ & $(10,20)$ & $(6,80)$ & $(6,46)$ & $(9,44)$ & $(6,35)$ & $(9,86)$ & $(8,54)$ & $(10,21)$ & $(8.75)$ \\
\hline \multirow{2}{*}{ Edad } & 42,16 & 39,95 & 40,05 & 36,80 & 39,22 & 39,94 & 40,79 & 39,43 & 41,16 & 39,49 \\
\hline & $(10,75)$ & $(9,75)$ & $(10,64)$ & $(8,60)$ & $(11,22)$ & $(10,95)$ & $(10,53)$ & $(10,27)$ & $(10,67)$ & $(10,21)$ \\
\hline
\end{tabular}

4 Divisiones 55-Servicios de Alojamiento y 56-Servicios de comida y bebida.

5 Comprendería todas las actividades entre los grupos 45 y 99, excepto los agregados en la hostelería, 55 y 56. 


\begin{tabular}{|c|r|r|r|r|r|r|r|r|r|r|}
\hline $\begin{array}{c}\text { Indefinidos a } \\
\text { tiempo com- } \\
\text { pleto }\end{array}$ & $\mathbf{0 , 8 3}$ & $\mathbf{0 , 7 6}$ & $\mathbf{0 , 5 4}$ & $\mathbf{0 , 6 1}$ & $\mathbf{0 , 6 9}$ & $\mathbf{0 , 6 5}$ & $\mathbf{0 , 7 3}$ & $\mathbf{0 , 5 7}$ & $\mathbf{0 , 7 4}$ & $\mathbf{0 , 6 0}$ \\
\cline { 2 - 10 } & $(0,37)$ & $(0,43)$ & $(0,50)$ & $(0,49)$ & $(0,46)$ & $(0,48)$ & $(0,44)$ & $(0,50)$ & $(0,44)$ & $(0,49)$ \\
\hline $\begin{array}{c}\text { Más de 19 } \\
\text { trabajadores }\end{array}$ & $\mathbf{0 , 7 9}$ & $\mathbf{0 , 7 7}$ & $\mathbf{0 , 6 6}$ & $\mathbf{0 , 6 0}$ & $\mathbf{0 , 7 9}$ & $\mathbf{0 , 8 1}$ & $\mathbf{0 , 8 2}$ & $\mathbf{0 , 8 3}$ & $\mathbf{0 , 7 9}$ & $\mathbf{0 , 8 2}$ \\
\cline { 2 - 11 }$y$ & $(0,41)$ & $(0,42)$ & $(0,48)$ & $(0,49)$ & $(0,40)$ & $(0,39)$ & $(0,39)$ & $(0,38)$ & $(0,41)$ & $(0,39)$ \\
\hline \multirow{2}{*}{$\begin{array}{c}\text { America } \\
\text { Latina }\end{array}$} & $\mathbf{0 , 0 1}$ & $\mathbf{0 , 0 2}$ & $\mathbf{0 , 0 4}$ & $\mathbf{0 , 0 2}$ & $\mathbf{0 , 0 7}$ & $\mathbf{0 , 0 7}$ & $\mathbf{0 , 0 2}$ & $\mathbf{0 , 0 3}$ & $\mathbf{0 , 0 2}$ & $\mathbf{0 , 0 3}$ \\
\cline { 2 - 10 }$y$ & $(0,11)$ & $(0,12)$ & $(0,20)$ & $(0,13)$ & $(0,26)$ & $(0,26)$ & $(0,14)$ & $(0,16)$ & $(0,14)$ & 0,16 \\
\hline \multirow{3}{*}{\begin{tabular}{c} 
Sobreedu \\
\cline { 2 - 10 }$y$
\end{tabular}} & $\mathbf{0 , 2 4}$ & $\mathbf{0 , 2 1}$ & $\mathbf{0 , 2 2}$ & $\mathbf{0 , 2 0}$ & $\mathbf{0 , 2 3}$ & $\mathbf{0 , 2 0}$ & $\mathbf{0 , 2 0}$ & $\mathbf{0 , 1 9}$ & $\mathbf{0 , 2 2}$ & $\mathbf{0 , 2 0}$ \\
\hline \multirow{2}{*}{ Infraedu } & $(0,43)$ & $(0,40)$ & $(0,42)$ & $(0,40)$ & $(0,42)$ & $(0,40)$ & $(0,40)$ & $(0,39)$ & $(0,41)$ & $(0,40)$ \\
\cline { 2 - 10 }$y$ & $(0,39)$ & $(0,40)$ & $(0,40)$ & $(0,47)$ & $(0,39)$ & $(0,32)$ & $(0,43)$ & $(0,41)$ & $(0,41)$ & $(0,41)$ \\
\hline
\end{tabular}

Notas: Entre paréntesis los valores de la desviación estándar de las variables. H= hombres y M= mujeres. Fuente: Encuesta de Estructura Salarial (EES- 2010). Elaboración propia.

Independientemente del sexo, la variable salario bruto medio por hora en la hostelería, presenta un valor promedio inferior al resto de actividades valoradas. No obstante, si se calculan las diferencias salariales, en términos absolutos, entre hombres y mujeres de todos los sectores, se apunta que la hostelería es, después de la construcción, la actividad económica donde la brecha es más reducida ${ }^{6}$.

La media de los años de estudios se observa superior para las mujeres en todos los sectores, con la excepción de la hostelería (8,17 años), donde el hombre invierte un poco más de tiempo (8,27 años). Al margen de este hecho, y de los bajos valores medios para esta variable en esta actividad, se observa, nuevamente, una reducida diferencia de los promedios entre hombres y mujeres. Por el contrario, la mayor diferencia de años de inversión educativa entre sexos se da en el sector de la construcción.

Si hay una actividad que destaque por la componente experiencia, esa es de nuevo, la hostelería. Los valores medios obtenidos en hombres (15,38 años) y mujeres $(18,59$ años), superan cómodamente el promedio nacional para cada sexo. Además, hay que mencionar que la mujer en la hostelería registra la media más elevada de años de experiencia, junto con el hombre en la construcción. Este hecho en el caso de la mujer podría estar relacionado con los bajos requerimientos educativos que generalmente han sido solicitados por la demanda laboral del sector hostelero. Dichos niveles reducidos habrían favorecido la temprana incorporación de la mujer al mercado laboral, acumulando años de experiencia.

Por lo que respecta a los años de antigüedad en el puesto de trabajo actual, destaca el valor medio de los hombres en la industria (12,30 años), muy superior a los obtenidos por sus congéneres en la construcción (5,44 años) u hostelería (7,76 años). A diferencia de la superioridad femenina en años medios de experiencia en la hostelería, la antigüedad, por su parte, queda relegada al promedio más reducido de toda la muestra (5,39 años).

En referencia a la edad, destaca su media elevada para el conjunto de la cohorte. Esta observación coincide con lo expuesto por Almodóvar et al. (2013), en relación a

6 Para la industria se ha calculado una diferencia de $3,22 € /$ hora; construcción $0,44 €$ /hora; hostelería $2,14 € /$ hora y para otros servicios $3,06 € /$ hora. 
que los trabajadores entre 31 y 54 años, son los que actualmente constituyen el grueso de la población activa en España. Los denominados "babyboomers", nacidos durante la explosión demográfica ocurrida entre 1958 y 1977.

En cuanto al tipo de contrato, destaca la proporción de más del $50 \%$ en todos los sectores y sexos de la modalidad contractual de duración indefinida y jornada completa. No obstante, también se aprecian diferencias significativas entre sexos. Al margen de la construcción, la proporción de mujeres contratadas con esta modalidad es inferior a la de los hombres. Esta elevada proporción en la hostelería encuentra su razón de peso en el uso de una modalidad especial de contrato fijo denominado fijo-discontinuo, que permite la interrupción de la relación laboral, como consecuencia del factor estacional (Muñoz-Bullón, 2009).

Para la variable representativa del tamaño de empresa, se observa una proporción que supera el $60 \%$ en todos los sectores, sin diferencias significativas entre sexos.

El porcentaje más elevado de trabajadores latinoamericanos entre todos los grupos de actividades se registra en la hostelería, donde alcanza el 7\%, tanto para el caso de las mujeres como para los hombres. Para García Ballesteros, Jiménez Basco y Redondo González (2009), esta actividad no solo constituye uno de los principales yacimientos de empleo para los inmigrantes latinoamericanos, sino que también contribuye a su visibilidad. De acuerdo con datos aportados por la Encuesta Nacional de Inmigrantes realizada por el INE en 2007, casi la mitad (47\%) de los trabajadores extranjeros en el sector de la hostelería, que llevaban más de 3 años de residencia en España, eran de origen latinoamericano.

Por último, en relación con las variables Sobreedu e Infraedu se observa que el desajuste educativo afecta, como mínimo, a un tercio de los ocupados hombres y mujeres de cada actividad. Los datos muestran que la mayor proporción del desajuste corresponde a los sobreeducados, salvo para la mujer en la construcción y para ambos sexos en otros servicios, cuya proporción de infraeducados es superior. Se aprecian diferencias mínimas en la proporción de hombres y mujeres afectados por el desajuste educativo. No obstante, se debe destacar la significativa diferencia en puntos porcentuales entre el porcentaje de ocupados/as infraeducados de la construcción (13 p.p.) y la hostelería (7 p.p.). Prestando mayor atención a esta última, se ha podido constatar, mediante la comparación de los resultados obtenidos para estas variables en este trabajo y los alcanzados por García-Pozo et al. (2012) para la misma base de datos en 2006, una reducción del desajuste educativo de la mujer en la hostelería española. Si en 2006, el porcentaje de ocupadas en hostelería afectadas por desajuste educativo se elevaba al 36\%, en 2010 se reduce al $32 \%$.

\section{RESULTADOS}

En la tabla 2 se presentan los resultados de las estimaciones de la ecuación [1], obtenidas a partir del método de mínimos cuadrados ordinarios. Concretamente se han realizado regresiones para las muestras de los sectores industrial, construcción, hostelería, otros servicios privados y para el conjunto de sectores de la economía española recogidos en la EES-2010, tanto para hombres como para mujeres, en cada uno de los sectores señalados. 


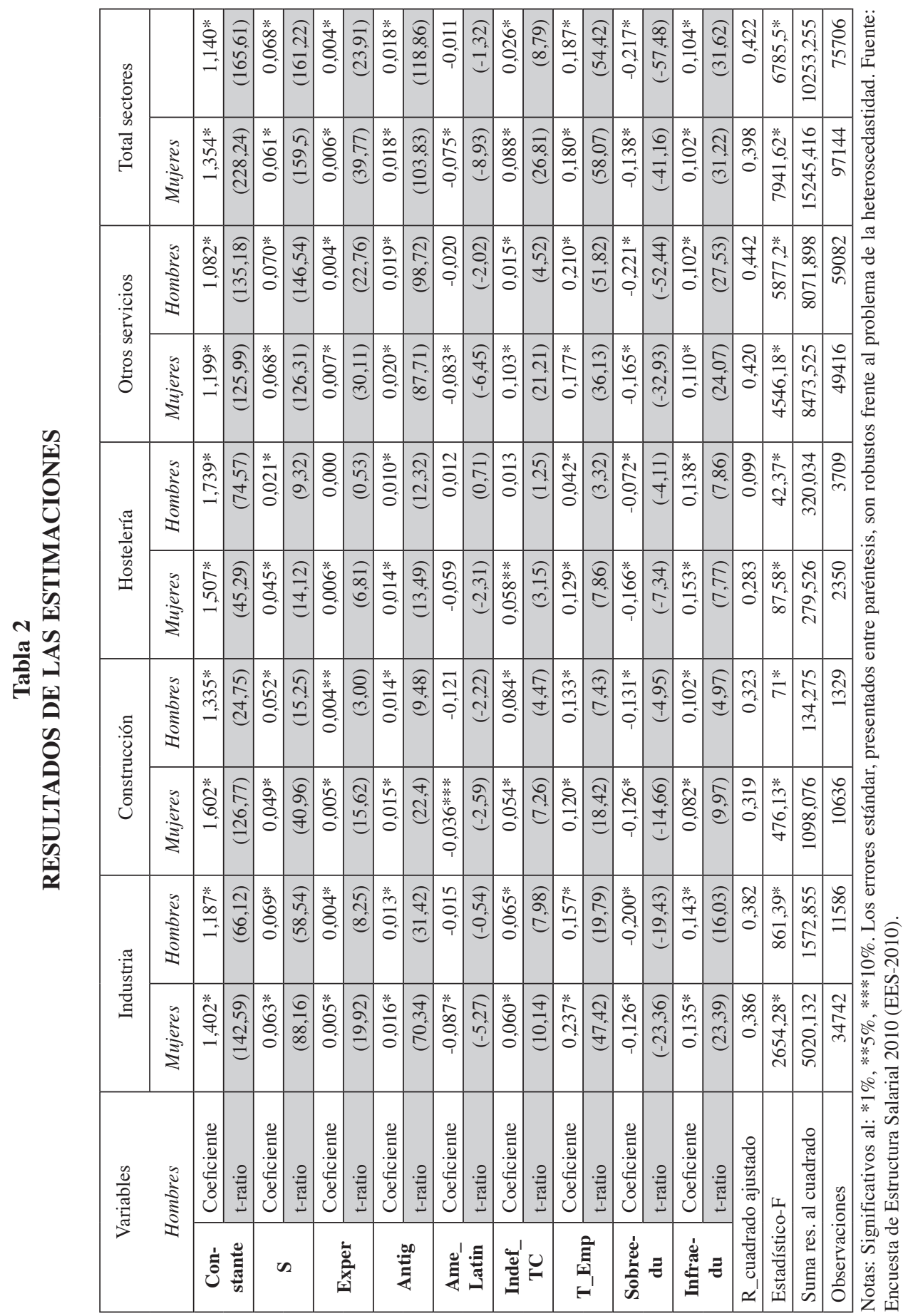


Es importante destacar que todas las funciones estimadas para cada sector de actividad y género presentan un valor estadísticamente significativo del test F. Por otra parte, la bondad del ajuste realizado en todas las regresiones estimadas queda corroborada por los valores calculados del estadístico $\mathrm{R}^{2}$-ajustado.

\subsection{Características de capital humano y personales}

Bajo condiciones de ceteris paribus la incidencia de la variable años medios de estudios $(S)$ sobre la variable dependiente $(W r)$, se traduce en una prima salarial. Es decir, por cada año adicional de estudios, el salario se incrementa en una tasa media indicada por el coeficiente. Este incremento en los salarios, se observa cuantitativamente superior en la mujer para todas las actividades valoradas, a excepción de la hostelería. A esta evidencia, se suma que la diferencia entre los rendimientos educativos de hombres y mujeres es la más alta en comparación con el resto de sectores valorados, alcanzando un diferencial negativo de las mujeres frente a los hombres del $114 \%$ por cada año adicional de estudio, cuando en el resto de sectores analizados ese diferencial nunca supera el $11 \%$. Y, a su vez, dichos rendimientos, en ambos sexos, son los más bajos de los obtenidos para la muestra $(2,1 \%$ en el caso de las mujeres y 4,5\% en el caso de los hombres). En línea con esto último, los resultados obtenidos en estudios previos para la hostelería y/o sector turístico en los que también se recogen las diferencias entre hombres y mujeres (García-Pozo et al., 2012; Lillo Bañuls y Casado Díaz, 2010, 2012; Sánchez-Ollero et al., 2014) y, en otros en los que no se tiene en cuenta (Fernández, Pena-Boquete y Pereira, 2009; García-Pozo, MarchanteMera y Sánchez-Ollero, 2011), coinciden en afirmar que las rentabilidades obtenidas para la educación en este subsector son inferiores a las alcanzadas por otros sectores privados. Los resultados aquí obtenidos siguen la tendencia y son comparables cuantitativamente con los estimados para el sector de la hostelería en estos trabajos que se citan.

En relación a la variable experiencia (Exper), si bien se observa que las estimaciones de los coeficientes son estadísticamente significativas (aunque con tasas muy reducidas) para ambos sexos en todos los sectores, no lo es para la mujer en la hostelería. Estos datos ponen de manifiesto la escasa importancia con la que el mercado laboral valora la experiencia previa al puesto de trabajo actual, siendo el componente del capital humano que menos rendimiento reporta a los trabajadores de la muestra.

Para todos los sectores implicados, los rendimientos de la variable antigüedad son estadísticamente significativos, con coeficientes positivos y superiores para el hombre. Por su parte, las mujeres obtienen rentabilidades inferiores, siendo la mujer en la hostelería la que registra la menor de las tasas. Esta evidencia también ha sido recogida por SánchezOllero et al. (2014) y García-Pozo et al. (2012).

Para finalizar con este grupo de componentes, merece la pena comentar que la variable (Ame_Latin) se presenta estadísticamente significativa únicamente para los hombres en todas las actividades, salvo para la hostelería. El coeficiente negativo obtenido para esta componente, se traduce en una penalización salarial. Esto es, un trabajador con nacionalidad latinoamericana percibe un salario en un porcentaje cuantitativamente inferior (indicado por el coeficiente) al que recibiría otro trabajador del mismo sector, pero de nacionalidad diferente. 


\subsection{Características del puesto de trabajo}

En referencia a la componente representativa del tipo de contrato, todos los coeficientes $^{7}$ son estadísticamente significativos y positivos para ambos sexos, salvo para la mujer en la hostelería. Además, se observa que son las mujeres las que obtienen un rendimiento salarial mayor por el disfrute de este tipo de contrato en el sector industrial y en la construcción, pero no sería el caso en el sector servicios. Concretamente y aplicando el método propuesto por Halvorsen \& Palmquist (1980) a la interpretación de los coeficientes estimados para las variables ficticias, en el sector de la hostelería los hombres que disfrutan de contratos indefinidos y a tiempo completo, obtienen una prima salarial del 5,97\% frente a aquellos que tienen contratos de otro tipo en este sector. Esta prima salarial no existe en este sector en el caso de las mujeres.

Los coeficientes estimados de la variable que recoge el tamaño empresarial son estadísticamente significativos y positivos en todos los sectores incluidos ya que, como se esperaba, las empresas de menos de 20 trabajadores muestran tendencia a remunerar menos a sus trabajadores. La mayor incidencia de esta componente sobre el salario se da en los hombres en la industria, donde por el hecho de trabajar en una empresa de un tamaño superior a 19 trabajadores, se obtiene una prima salarial del 26,74\%. Muy lejos de este último rendimiento, se encuentra el 4,29\% de la mujer en la hostelería.

El signo positivo de esta componente es una evidencia de la asociación directa que existe entre el mayor tamaño empresarial con unas retribuciones más elevadas (Brown \& Medoff, 1989). En el contexto del sistema de negociación colectiva en España, los salarios sectoriales aumentan conforme mayor es el peso de las grandes empresas y al incrementarse el número de trabajadores con convenios (Aláez Aller, Longás García y Ulibarri Arce, 2003), los cuales suelen tener carácter específico y están orientados fundamentalmente a la mejora de las condiciones laborales básicas ya pactadas en los convenios de ámbito territorial.

Por último, se observan los efectos de las variables representativas del desajuste educativo (Sobreedu e Infraedu). Por un lado, la incidencia de la sobreeducación sobre el salario se traduce en una penalización nada desdeñable para la mujer. En otras palabras, las trabajadoras que estén sobreeducadas para un puesto de trabajo (tienen más educación de la que se requiere para su desempeño), obtienen una remuneración menor que otras con el mismo nivel educativo, pero cuyo puesto requiere precisamente del nivel que poseen (están adecuadamente educadas). Para la hostelería, los valores del coeficiente de la variable Sobreedu para el hombre y la mujer recogen dos extremos. Mientras que la penalización asociada a la mujer es la menor de todas $(-6,94 \%)$, entre las homónimas del resto de actividades, la del hombre $(-15,30 \%)$ es la más elevada. Para completar el análisis de las diferencias de género, los sectores de la industria y construcción muestran la menor penalización salarial para el hombre sobreeducado $(-11,84 \%)$, mientras que la mujer ocupada en otros servicios, es la más afectada por este desajuste $(-19,82 \%)$ en toda la muestra.

Por otro lado, el valor positivo de los coeficientes obtenidos para la variable infraeducación indica que: un trabajador/a con un nivel educativo inferior al que se requiere

7 Valor del coeficiente estimado una vez interpretado $=\left(e^{\beta \mathrm{i}}-1\right)^{*} 100$ 
para el puesto de trabajo que ocupa, recibe un salario mayor que otro trabajador/a con el mismo nivel educativo, pero que está empleado en un puesto para el que se requiere el nivel que posee (adecuadamente educado). En cuestión de género y sector, son las mujeres del sector industrial $(15,37 \%)$ y los hombres en la hostelería $(16,53 \%)$, los más favorecidos económicamente por esta condición.

\section{CONCLUSIONES}

Bajo una perspectiva de género, este trabajo aporta evidencia empírica de la incidencia de varios factores personales y laborales sobre el salario de los trabajadores/as del subsector de la hostelería española, comparado con otros sectores de actividad. En un contexto en el que el turismo es un sector de vital importancia para la economía española, junto a la presencia mayoritaria de mujeres ocupadas en la industria hostelera y, todo ello, condicionado por la generalizada precariedad salarial de la mujer, hacen que esta investigación adquiera especial interés en el estudio de las diferencias salariales entre hombres y mujeres españoles. El análisis efectuado se basa en la estimación de funciones de salarios diferenciadas por sexos y actividades económicas, con el objetivo de verificar la existencia de desigualdades salariales. El empleo de microdatos emparejados empresa-trabajador, favorece la inclusión en el modelo económico de variables tanto de capital humano como de otras relacionadas con las características del trabajador y de su puesto de trabajo. De esta manera, las estimaciones obtenidas para hombres y mujeres ocupados en las actividades de la industria, construcción, hostelería y otros servicios, permiten cuantificar y comparar el rendimiento salarial de las variables analizadas.

Los resultados indican que los rendimientos de la educación para los trabajadores de la hostelería española son destacablemente inferiores, y con mayor intensidad en la mujer, a los del resto de actividades económicas analizadas. Este resultado, unido al elevado porcentaje de trabajadores que están sobreeducados para su puesto de trabajo (más del $50 \%$ de ocupados de la muestra) y a la tardía incorporación en nuestro país de las enseñanzas del turismo al catálogo de los estudios universitarios (López Bonilla y López Bonilla, 2004), sugiere, cuanto menos, la necesidad de un reflexivo replanteamiento sobre la respuesta del sistema educativo a la demanda real del mercado laboral turístico.

En referencia a las variables experiencia y antigüedad en el puesto de trabajo, los resultados demuestran su escasa e incluso nula influencia sobre los salarios, especialmente para la mujer en la hostelería. En un contexto actual, las mujeres siguen asumiendo la gran mayoría de las labores de cuidado de los hijos y las tareas domésticas no remuneradas (Organización Internacional del Trabajo, 2016). En consecuencia, la probabilidad de abandono temporal o definitivo de su puesto de trabajo es más elevada, abreviando su permanencia en la empresa. De ahí que las primas salariales para ambas variables sean más reducidas o inexistentes. Estos mismos resultados son observados para la modalidad de contrato y el tamaño del establecimiento.

Por su parte, de estas dos últimas características señaladas, relacionadas con el puesto de trabajo, cabe destacar sus primas salariales asociadas. En el caso concreto de la dimensión del establecimiento, se observa el efecto salario-tamaño en aquellos sectores que cuentan con empresas más grandes (en nuestra muestra, de más de 19 trabajadores). En 
concreto, la industria y otros servicios, registran las mayores primas salariales, mientras que en la hostelería se reducen. De acuerdo con el Instituto Nacional de Estadística (2016), a 1 de enero de 2016, los mayores porcentajes de empresas pequeñas se concentraron en el sector resto de servicios (el 84,3\% tenía dos o menos asalariados).

A partir de este trabajo, se ha podido constatar que la diferencia salarial por razón de género es una realidad en todas las actividades valoradas. No obstante, sorprende que sea la hostelería junto con la construcción, las actividades donde la brecha salarial es más pequeña. Por otro lado, no resulta tan extraño que sea la hostelería, la actividad que registra el nivel medio de salarios más bajo, siendo la mujer la más afectada en este sentido. Como se ha puesto de manifiesto anteriormente, la gran carga asumida por la mujer en el cuidado del hogar y de los hijos, limita potencialmente su capacidad para emplearse y/o promocionarse en su carrera profesional. En ese sentido, se han visto relegadas a concentrarse en sectores u ocupaciones peor remuneradas.

En completa consonancia con la Organización Internacional del Trabajo (2016), en la explicación de las desigualdades salariales no sólo basta considerar variables presumibles de estimarles un coeficiente, sino que también hay que valorar otras causas. A tener en cuenta, por ejemplo, están la infravaloración de los trabajos realizados por las mujeres, las calificaciones requeridas en las ocupaciones dominadas por éstas o la práctica de la discriminación. Actualmente, es un hecho contrastable la apuesta por políticas de igualdad de género y de reducción de la brecha salarial. Aunque se han realizado ciertos progresos en este último aspecto, el logro se atribuye especialmente a medidas de política explícitas encaminadas a hacer frente a los desequilibrios de género en el mercado laboral, más que a la mejora general del nivel de vida.

Por tanto, la mitigación de esta realidad no pasa por reequilibrar los niveles salariales en un período de corto a medio plazo, sino que implica atacar el problema desde su raíz. Las implicaciones resultan, cuanto menos, ambiciosas, pues supone avanzar hacia el cambio en los preceptos culturales y sociales que secundan y reafirman la existencia de salarios diferentes para hombres y mujeres. La comprensión y materialización en apuestas claras, sobre todo en las generaciones venideras, podría ser clave para la reducción de las desigualdades de género en cualquier campo.

\section{BIBLIOGRAFÍA}

ALÁEZ ALLER, R., LONGÁS GARCÍA, J. C. y ULIBARRI ARCE, M. (2003): «Diferencias salariales en España: un análisis sectorial/regional», Investigaciones Regionales, vol. 3, pp. 5-24.

ALMODÓVAR, A., GALIANA, L., GÓMEZ-CANO, M. y MUÑOZ, M. (2013): Análisis del mercado laboral, condiciones de trabajo y siniestralidad: una perspectiva según la edad, Instituto Nacional de Seguridad e Higiene en el Trabajo. Disponible en: http:// www.oect.es/Observatorio/5 Estudios tecnicos/Otros estudios tecnicos/Publicado/ Ficheros/INFORME sobre la edad (INSHT 2013).pdf

ANKER, R. (1997): «La segregación profesional entre hombres y mujeres. Repaso de las teorías», Revista Internacional del Trabajo, n 116 , vol. 3, pp. 344-370. 
ARRAZOLA VACAS, M., y HEVIA PAYÁ, J. (2006): «Gender differentials in returns to education in Spain», Education Economic, n ${ }^{\circ}$ 14, vol. 4, pp. 469-486.

BAUM, T. (2013): International perspectives on women and work in hotels, catering and tourism (Sectoral Activities Department No. 289). Geneva, International Labour Office.

BECKER, G.S. (1957): The economics of discrimination. The University of Chicago Press.

BECKER, G.S. (1964): Human Capital: a theoretical and empirical analysis with special reference to education. Chicago: University of Chicago Press.

BLANCO, J.M. (1997): «Comentarios acerca del desajuste educativo en España», Papeles de Economía Española, vol. 72, pp. 275-291.

BLAU, F. (1972): «Women's place in the labor market», Annals of Tourism Research, vol. 62, pp. 161-166.

BLINDER, A. (1973): «Wage discrimination: reduced form and structural estimates», Journal of Human Resources, $\mathrm{n}^{\circ}$ 8, pp. 436-455.

BROWN, C. y MEDOFF, J. (1989): «The employer size-wage effect», The Journal of Political Economy, n95, vol. 5, pp. 1027-1059.

BYRNE SWAIN, M. (1995): «Gender in tourism», Annals of Tourism Research, $\mathrm{n}^{\circ} 22$, vol. 2, pp. 247-266.

CAMPOS-SORIA, J.A., MARCHANTE-MERA, A. y ROPERO-GARCÍA, M.A. (2011): «Patterns of occupational segregation by gender in the hospitality industry», International Journal of Hospitality Management, $\mathrm{n}^{\circ}$ 30, vol. 1, pp. 91-102.

CAMPOS-SORIA, J.A., ORTEGA-AGUAZA, B. y ROPERO-GARCÍA., M.A. (2010): «Diferentes patrones de segregación de género y diferencia salarial entre hombres y mujeres en la hostelería», Estudios de Economía Aplicada, no 28, vol. 1, pp. 1-30.

CAMPOS-SORIA, J.A., ORTEGA-AGUAZA, B. y ROPERO-GARCÍA, M.A. (2009): «Gender segregation and wage difference in the hospitality industry», Tourism Economics, $\mathrm{n}^{\circ} 15$, vol. 4, pp. 847-866.

CASADO-DÍAZ, J.M. y SIMÓN, H. (2016): «Wage differences in the hospitality sector», Tourism Management, ${ }^{\circ}$ 52, pp. 96-109.

DE CABO SERRANO, G. y GARZÓN, M.J. (2007): Diferencia y discriminación salarial por razón de sexo, Instituto de la Mujer, Madrid.

DE LA RICA, S. y UGIDOS, A. (1995): « ¿Son las diferencias salariales determinantes de las diferencias salariales observadas entre hombres y mujeres?, Investigaciones Económicas, n $^{\circ}$ 19, vol. 3, pp. 395-414.

EUROSTAT (2016): «Gender pay gap statistics». En línea: http://ec.europa.eu/eurostat/ statistics-explained/index.php/Gender_pay_gap_statistics [12 de agosto de 2016].

FERNÁNDEZ, M., PENA-BOQUETE, Y. y PEREIRA, X. (2009): «Labor conditions in the Spanish hotels and restaurants industry», Tourism Analysis, ${ }^{\circ}$ 14, vol. 3, pp. 293-312.

FERREIRA FREIRE GUIMARAES, C. R. y SILVA, J.R. (2016): «Pay gap by gender in the tourism industry of Brazil», Tourism Management, $\mathrm{n}^{\circ}$ 52, pp. 440-450.

FLEMING, S.S. (2015): «Dejà vu? An updated analysis of gender pay gap in the U.S. hospitality sector», Cornell Hospitality Quaterly, n 52, vol. 2, pp. 180-190. 
GARCÍA BALLESTEROS, A., JIMÉNEZ BASCO, B. y REDONDO GONZÁLEZ, A. (20n09): «La inmigración latinoamericana en España en el siglo XXI», Investigaciones Geográficas, Boletín Del Instituto de Geografía, vol.70, pp. 55-70.

GARCÍA-POZO, A., CAMPOS-SORIA, J.A., SÁNCHEZ-OLLERO, J.L. y MARCHANTE-LARA, M. (2012): «The regional wage gap in the Spanish hospitality sector based on a gender perspective», International Journal of Hospitality Management, n³1, vol. 1, pp. 266-275.

GARCÍA-POZO, A., MARCHANTE-MERA, A. y SÁNCHEZ-OLLERO, J.L. (2011): «Occupational differences in the return on human capital in the Spanish travel agency and hospitality industries», Tourism Economics, n 17 , vol. 6, pp. 1325-1345.

HALVORSEN, R. y PALMQUIST, R. (1980): «The interpretation of dummy variables in semilogarithmic equations», American Economic Review, n 70, vol. 3, p. 475.

HERNÁNDEZ MARTÍNEZ, P. J. (1995): «Análisis empírico de la discriminación salarial de la mujer en España», Investigaciones Económicas, nº 19, vol. 2, pp. 195-215.

INSTITUTO NACIONAL DE ESTADÍSTICA (2016): «Estructura y dinamismo del tejido empresarial en España. Directorio Central de Empresas (DIRCE)».www.ine.es

KIKER, B., SANTOS, M. y MENDES DE OLIVEIRIA, M. (1997): «Overeducation and undereducation: evidence for Portugal», Economics of Education Review, nº 16, vol. 2, pp. 111-125.

KINNARD, V. y HALL, D. (1994): Tourism: a gender analysis. Wiley: Chichester.

LEE, C.K. y KANG, S. . (1998): «Measuring earnings inequality and median earnings in the tourism industry», Tourism Management, $\mathrm{n}^{\circ}$ 19, vol. 4, pp. 341-348.

LILLO BAÑULS, A. (2009): «Capital humano y rendimiento educativo en turismo», Estudios Turísticos, vol. 179, pp. 7-26.

LILLO BAÑULS, A. y CASADO DÍAZ, J.M. (2010): «Rewards to education in the tourism sector: one step ahead», Tourism Economics, $\mathrm{n}^{\circ}$ 16, vol, 1, pp. 11-23.

LILLO BAÑULS, A. y CASADO DÍAZ, J.M. (2011): «Capital humano y turismo: rendimiento educativo, desajuste y satisfacción laboral», Estudios de Economía Aplicada, $\mathrm{n}^{\circ}$ 29, vol. 3, pp. 755-780.

LILLO BAÑULS, A. y CASADO DÍAZ, J.M. (2012): «Individual returns to education in the Spanish tourism sector during the economic crisis», Tourism Economics, $\mathrm{n}^{\circ} 18$, vol. 6, pp. 1229-1249.

LILLO BAÑULS, A. y RAMÓN RODRÍGUEZ, A.B. (2005): «Returns on education in the Spanish tourism labour market», Tourism Economics, $\mathrm{n}^{\circ} 11$, vol. 1, pp. 59-62.

LÓPEZ BONILLA, J. M. y LÓPEZ BONILLA, L.M. (2004): «Evolución y perspectivas del enfoque interdisciplinario en el estudio del turismo», Estudios Turísticos, vol. 160, pp. 31-44.

LUNDBERG, S. y STARZ, R. (1983): «Private discrimination and social intervention in competitive labor markets», American Economic Review, n 16, vol. 1, pp. 340-347.

MALKIEL, B. y MALKIEL, J. (1973): «Male-female pay differentials in professional employment», American Economic Review, n 63, vol. 4, pp. 693-705.

MARCHANTE, A. J., ORTEGA, B. y PAGÁN, R. (2005): «Educational mismatch and wages in the hospitality sector», Tourism Economics, n' ${ }^{\circ} 11$, vol. 11, pp. 103-117. 
MARCHANTE MERA, A., ORTEGA AGUAZA, B. y SÁNCHEZ OLLERO, J.L. (2004): «Desajuste educativo y movilidad laboral de los trabajadores de hostelería en Andalucía», Revista de Estudios Regionales, nº 69, pp. 77-88.

MARRERO RODRÍGUEZ, J.R. (2015): «La sobrecualificación en el sector turístico : el caso español (1987-2011)», Cuaderno de Relaciones Laborales, $\mathrm{n}^{\circ}$ 33, vol. 1, pp. 149-168.

MCCONNEL, C. R. y BRUE, S.L. (1996): Economía laboral (Trad.E. Rabasco) (4.a ed.). Madrid: McGraw-Hill (Original de 1996).

MINCER, J. (1974): Schooling, experience and earnings. New York: Columbia University Press.

MOLTÓ, M.L. (1984): «Estudio empírico de la discriminación de la mujer en el mercado de trabajo académico», Estadística Española, vol. 102, pp. 105-128.

MUÑOZ-BULLÓN, F. (2009): «The gap between male and female pay in the Spanish tourism industry», Tourism Management, $\mathrm{n}^{\circ}$ 30, vol. 5, pp. 638-649.

NICKSON, D. (2007): Human resource management for the hospitality and tourism industries. Oxford: Butterworth-Heinemann.

OAXACA, R. (1973): «Male-female wage differentials in urban labor markets», International Economic Review, no 14, vol. 3, pp. 639-709.

ORGANIZACIÓN INTERNACIONAL DEL TRABAJO (2016): «Las mujeres en el trabajo. Tendencias de 2016». Ginebra.

ORGANIZACIÓN MUNDIAL DEL TURISMO (2016). «Panorama OMT del turismo internacional».

PEINADO, A. (1988): La discriminación de la mujer en el mercado de trabajo español: una aproximación empírica a la discriminación salarial. Ministerio de Trabajo y Seguridad Social, Centro de Publicaciones, Madrid.

PONZELLINI, A. M., AUMAYR, C. y WOLF, F. (2010). Addressing the gender pay gap: Government and social partner actions, Eurofound. En línea: http://www.eurofound. europa.eu/publications/report/2010/working-conditions-industrial-relations/addressing-the-gender-pay-gap-government-and-social-partner-actions [2 de julio de 2016].

PRIETO, J. (1995): Discriminación salarial y movilidad laboral. Tesis doctoral no publicada. Universidad de Oviedo.

RAMOS, V., REY-MAQUIEIRA, J. y TUGORES, M. (2002): «Análisis empírico de discriminación por razón de género en una economía especializada en turismo», Annals of Tourism Research En Español, n ${ }^{\circ}$ 4, vol. 1, pp. 239-258.

RIBAUD, M. y HERNÁNDEZ, F. (1989). Un análisis de la discriminación salarial de las mujeres en España, Instituto de la Mujer, Ministerio de Asuntos Sociales, Madrid.

RUEDA NARVÁEZ, M.F. (2010): Discriminación salarial por razón de género y capital humano. Pamplona: Civitas.

SÁNCHEZ-OLLERO, J.L., CAMPOS-SORIA, J.A. y GARCÍA-POZO, A. (2014): «The labour market in the Spanish hospitality industry: an overview from a gender perspective», Revista Turismo \& Desenvolvimiento, vol. 21/22.

SCHULTZ, T. W. (1961): «Investment in human capital», American Economic Review, $\mathrm{n}^{\mathrm{o}} 51, \mathrm{pp} .1-17$. 
SELVA SEVILLA, C. (2004). El capital humano y su contribución al crecimiento económico, un análisis para Castilla-La Mancha. Ediciones de la Universidad de Castilla-La Mancha: Cuenca.

SIMÓN, H. (2006): «Diferencias salariales entre hombres y mujeres en España: una comparación internacional con datos emparejados empresa-trabajador», Investigaciones Económicas, $\mathrm{n}^{\mathrm{o}}$ 30, vol. 1, pp. 55-87.

SINCLAIR, T. (1997): Issues and theories of gender and work in tourism research. In T. Sinclair (Ed.), Gender work and tourism. London: Routledge.

SPARROWE, R. T. e IVERSON, K.M. (1999): «Craks in the glass ceiling? An empirical study of gender differences in income in the hospitality industry», Journal of Hospitality \& Tourism Research, $\mathrm{n}^{\mathrm{O}} 23$, vol. 1, pp. 4-20.

STRAUSS, H. y MAISONNEUVE, C. (2007): «The wage premium on tertiary education: new estimates for 21 OECD countries», OECD Journal: Economic Studies, vol. 2009.

THUROW, L.C. (1975): Generating inequality: mechanisms of distribution in the U.S. economy. New York: Basic Books.

ULIBARRI ARCE, M. (2003): «Diferencias salariales entre los sectores público y privado por género, escolaridad y edad. El caso de España», El Trimestre Económico, vol. 70, pp. 233-253.

VERDUGO, R. y VERDUGO, N.T. (1989): «The impact of surplus schooling on earnings: some additional findings, Journal of Human Resources, $\mathrm{n}^{\circ}$ 24, pp. 629-695.

WILKINSON, P.F. y PRATIWI, W. (1995): «Gender and tourism in an Indonesian village», Annals of Tourism Research, $\mathrm{n}^{\circ} 22$, vol. 2, pp. 283-299. 